\title{
X-ray lasers: the evolution from Star Wars to the table-top
}

Joseph Nilsen

Joseph Nilsen, "X-ray lasers: the evolution from Star Wars to the table-top," Proc. SPIE 11886, International Conference on X-Ray Lasers 2020, 1188604 (8 July 2021); doi: 10.1117/12.2593255

SPIE. Event: XVII International Conference on X-Ray Lasers, 2020, Online Only 


\title{
X-ray lasers: The evolution from Star Wars to the table-top
}

\author{
Joseph Nilsen \\ Lawrence Livermore National Laboratory, Livermore, CA 94551
}

\begin{abstract}
In this paper we tell the story of the X-ray laser, which began as an attempt to extend optical lasers to shorter wavelengths in the 1970's. Research took off in the 1980's driven by the rivalry between the United States and Soviet Union in their quest to create a "Star Wars" laser shield against ICBM's. At the same time large inertial fusion confinement (ICF) lasers, such as Novette and Nova at LLNL, were able to create the first laboratory X-ray lasers in Ne-like Se at 20.6 and $20.9 \mathrm{~nm}$ using $2 \mathrm{~kJ}$ of energy in a $0.5 \mathrm{~ns}$ pulse. These large ICF lasers could only be fired every few hours and used very thin expensive exploding foils as the targets. With the demonstration of the pre-pulse technique at Nova, where a small pulse of a few joules heated a solid target and create a pre-plasma that was then be heated by a second large pulse to create the lasing conditions, X-ray lasers started working robustly at many laser facilities around the world. The advent of high repetition rate psec lasers combined with the pre-pulse technique and grazing incident geometry opened the door to the many table-top X-ray lasers today which can be driven by less than $1 \mathrm{~J}$ of energy and operate at $100 \mathrm{~Hz}$ repetition rates. This has opened many new research opportunities for scientific research using X-ray lasers.
\end{abstract}

Keywords: X-ray laser, Star Wars, Nova, Novette, Comet, X-ray optics

\section{EARLY DAYS OF X-RAY LASER}

As soon as lasers were invented scientists were thinking of how to achieve shorter wavelengths and extend optical lasers into the X-ray regime [1-5]. The mid 1970's saw many papers written about X-ray lasers. One paper from Lawrence Livermore National Laboratory (LLNL) published in Physics Today was written by two early pioneers, George Chapline and Lowell Wood [6]. That paper looked at many of the then current X-ray laser ideas and suggested that X-ray lasers would have far-reaching impacts on biology, chemistry and crystallography and could one day be used to resolve the structure of molecules.

The worldwide community was already conducting laser-driven plasma experiments with the hope of producing an Xray laser. The Physics Today paper suggested that one of the most promising laser schemes was to lase at $0.5 \mathrm{~nm}$ on the $3 \mathrm{~d}$ to $2 \mathrm{p}$ transition in hydrogen-like krypton, which is a one electron system. This is a high- $Z$ analogous to the hydrogenlike carbon (C VI) laser demonstrated a decade later at $18.2 \mathrm{~nm}$ [7] at the Princeton Plasma Physics Laboratory. Chapline and Wood suggested that a series of psec lasers could be set up to illuminate a cylindrical target from the side with a timing offset between the lasers that would create a traveling wave geometry so that the plasma was illuminated along its length as the X-ray laser beam propagated down the length of the gain medium. They acknowledged that current lasers could not yet achieve these goals. The cover page of the Physics Today article shows the JANUS target chamber and explained that X-ray laser experiments were planned using this large ICF laser. Ironically, more than 20 years later a low energy beam of JANUS was used to provide the pre-pulse for the first LLNL experiments to use the combination of a nsec pre-pulse and psec main pulse to drive an X-ray laser with less than 10 joules of energy.

As several research groups started seeing signs of gain on various X-ray laser transitions, one of the first published papers that looked promising was the 1977 paper [3] by Ilyukhin and colleagues from the P. N. Lebedev Physical Institute who saw a signal near $60.0 \mathrm{~nm}$ that they thought could be coming from a $3 p-3 \mathrm{~s}$ neon-like calcium transition. Many papers were written about potential neon-like X-ray lasers $[4,5,8,9]$ on these transitions and seven years later in 1984 LLNL demonstrated the first laboratory X-ray laser in neon-like selenium on these transitions [8]. It should be noted that today, with the advent of the pre-pulse technique [10], the $3 p{ }^{1} \mathrm{~S}_{0}-3 \mathrm{~s}{ }^{1} \mathrm{P}_{1}$ line is the dominant laser line for neon-like systems, as was originally predicted by Vinogradov and his colleagues from the P. N. Lebedev Physical Institute back in 1977 [4]. This line is created by ionizing the laser medium to the ground state of the neon-like ionization stage which 
means there are 10 bound electrons filling the $\mathrm{K}$ and $\mathrm{L}$ shells. When the material is sufficiently hot there is a strong monopole collisional excitation from the $1 s^{2} 2 s^{2} 2 p^{6}{ }^{1} S_{0}$ ground state to the $1 s^{2} 2 s^{2} 2 p^{5} 3 p{ }^{1} S_{0}$ excited state. This $1 s^{2} 2 s^{2} 2 p^{5} 3 p$ ${ }^{1} \mathrm{~S}_{0}$ state is metastable and decays via the $1 \mathrm{~s}^{2} 2 \mathrm{~s}^{2} 2 \mathrm{p}^{5} 3 \mathrm{p}^{1} \mathrm{~S}_{0}-1 \mathrm{~s}^{2} 2 \mathrm{~s}^{2} 2 \mathrm{p}^{5} 3 \mathrm{~s}^{1} \mathrm{P}_{1}$ to create lasing. The lower laser state then decays back to the ground state. For simplicity people usually leave out the core electrons and refer to the $3 \mathrm{p}^{1} \mathrm{~S}_{0}-3 \mathrm{~s}{ }^{1} \mathrm{P}_{1}$ transition. A nice review of the early history of X-ray lasers can be found in Ray Elton's book in Ref. 5. In this paper we focus on the pre-dominant neon-like and nickel-like schemes but there are other laser schemes which have lased such as recombination, capillary discharge, and optical field ionization (OFI) that all are discussed in Ref. 11.

\section{LEGACY OF STAR WARS ERA}

In the early 1980's the news media took notice of the X-ray laser effort because of the missile defense application that would eventually be the primary motivation for X-ray laser research in the United States. In a 1981 Aviation Week article [12] Clarence Robinson wrote that scientists from LLNL had made a breakthrough in laser technology by demonstrating a compact laser device pumped by X-rays from a small nuclear detonation. President Reagan's "Star Wars" speech on March 23, 1983 challenged the scientific community to find a defense against nuclear-tipped ballistic missiles as shown in Fig. 1. With the creation of the Strategic Defense Initiative (SDI) X-ray laser research became the biggest research effort at LLNL and funded most X-ray laser research and much of the atomic physics research in the United States. This made the early 1980's a very exciting time for the X-ray laser research. As part of this program [13-15] LLNL succeeded in demonstrating the first X-ray laser in the early 1980's using a nuclear explosion to drive the X-ray laser.

The 1997 article [16] written by E. N. Avrorin and colleagues at Chelyabinsk-70 shows that the Soviet Union also took a strong interest in the X-ray laser. In this article they describe how the Soviet Union initiated a program to understand $\mathrm{X}$-ray laser physics and evaluate the feasibility of making an X-ray laser with the characteristics reported in the Aviation Week article. This article reports that experiments were carried out in 1987 using nuclear explosions that successful produced X-ray lasers with about $20 \mathrm{~kJ}$ of output at $39 \AA$ and $100 \mathrm{~kJ}$ of output at a wavelength of $28 \AA$. Clearly the

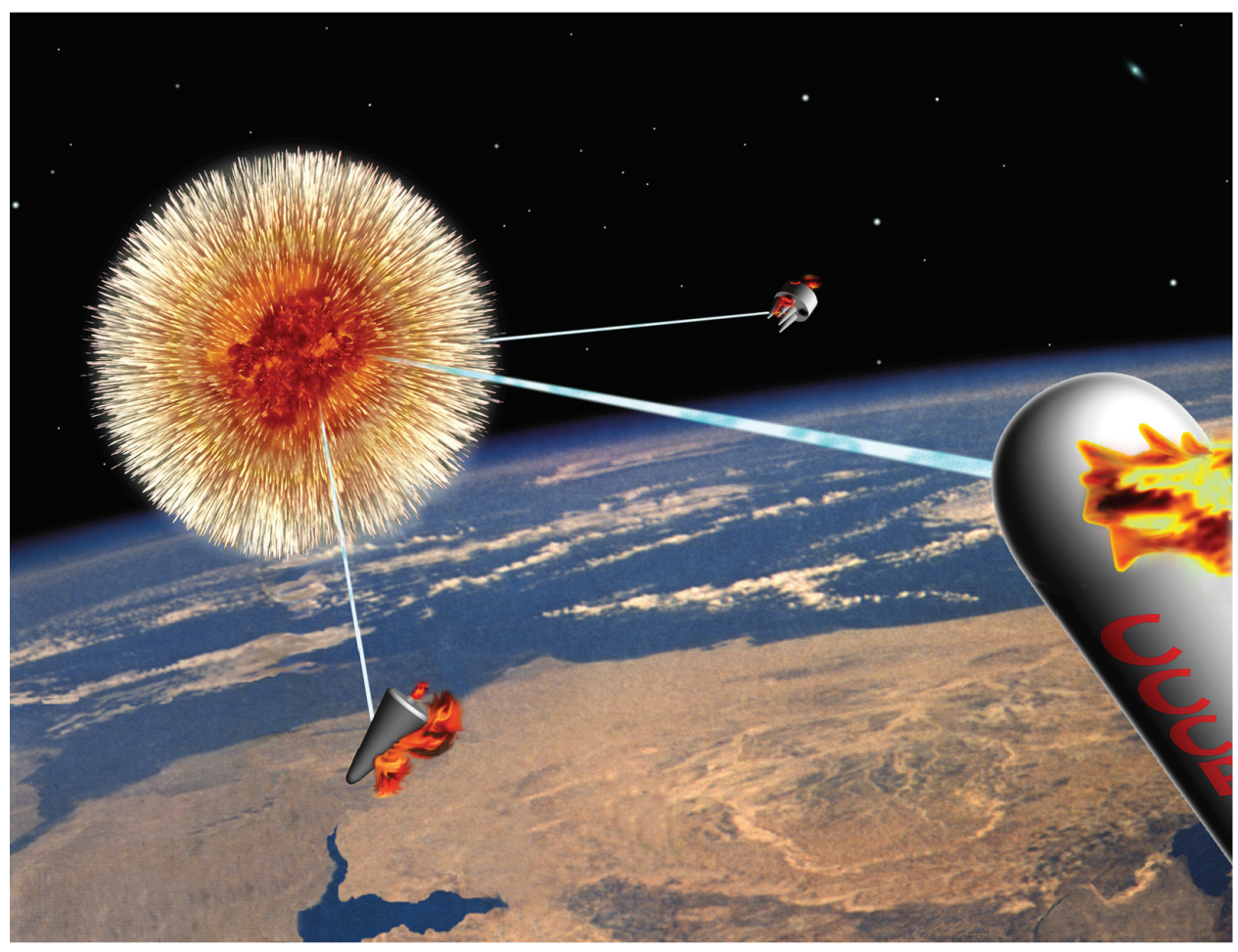

Fig. 1. Conceptual plan for using an X-ray laser for missile defense against the booster, warhead bus, or individual re-entry vehicle (RV) during different phases of an ICBM flight trajectory. 
competition between the United States and the Soviet Union was a significant driver in the development of the X-ray laser.

With the end of nuclear testing in 1992 the Star Wars X-ray laser program at LLNL ended. While the technical legacy of the Star Wars X-ray laser program is still classified, details of the historical legacy can be found in Refs. $13-15$. To summarize, some of the achievements of the X-ray laser program during this period include the following: the first laboratory X-ray laser; super light aerogels; advanced plasma kinetics and atomic physics codes; greatly improved diagnostics; the electron-beam ion trap (EBIT) for atomic physics measurements; new digital mammography techniques; high-resolution X-ray microscopes using zone plates; advanced multi-layer X-ray optics; and the demonstration of the world's shortest wavelength, highest energy laser.

\section{NOVA XRAY LASER}

At LLNL the first laboratory X-ray laser was demonstrated $[8,9]$ at the Novette laser facility in 1984 on a pair of $3 p$ 3 s neon-like selenium $(Z=34)$ lines at 20.63 and $20.96 \mathrm{~nm}$ using a thin exploding foil target, shown in Fig. 2, that consisted of $75 \mathrm{~nm}$ of Se coated on $150 \mathrm{~nm}$ of plastic that was illuminated from each side with a $450 \mathrm{ps}, 1 \mathrm{~kJ}$ pulse of frequency doubled light at $0.53 \mu \mathrm{m}$. The laser was focused to a $200 \mu \mathrm{m}$ wide by $1.1 \mathrm{~cm}$ long beam to create a long slender plasma that would preferentially amplify the X-ray down the $1.1 \mathrm{~cm}$ long axis. The conditions in the lasing plasma were estimated to have an electron temperature of about $1 \mathrm{keV}$ and an electron density of $10^{21} \mathrm{~cm}^{-3}$. The original gain was measured to be $5.5 \mathrm{~cm}^{-1}$, which gave a gain-length product of 6.5. This ushered in a decade of laser experiments at the Nova 2-beam facility that was funded by the Defense Sciences Department to help understand basic X-ray laser physics. Novette was a neodymium glass laser with wavelength $1.053 \mu \mathrm{m}$ that was the proof of concept for the 10-beam Nova laser facility built for ICF research. A separate 2-beam target chamber was built at the Nova laser facility for X-ray laser research and operated with frequency doubled light while the main ICF laser using frequency-tripled light. Figure 3 shows the enormous size of the Nova laser facility.

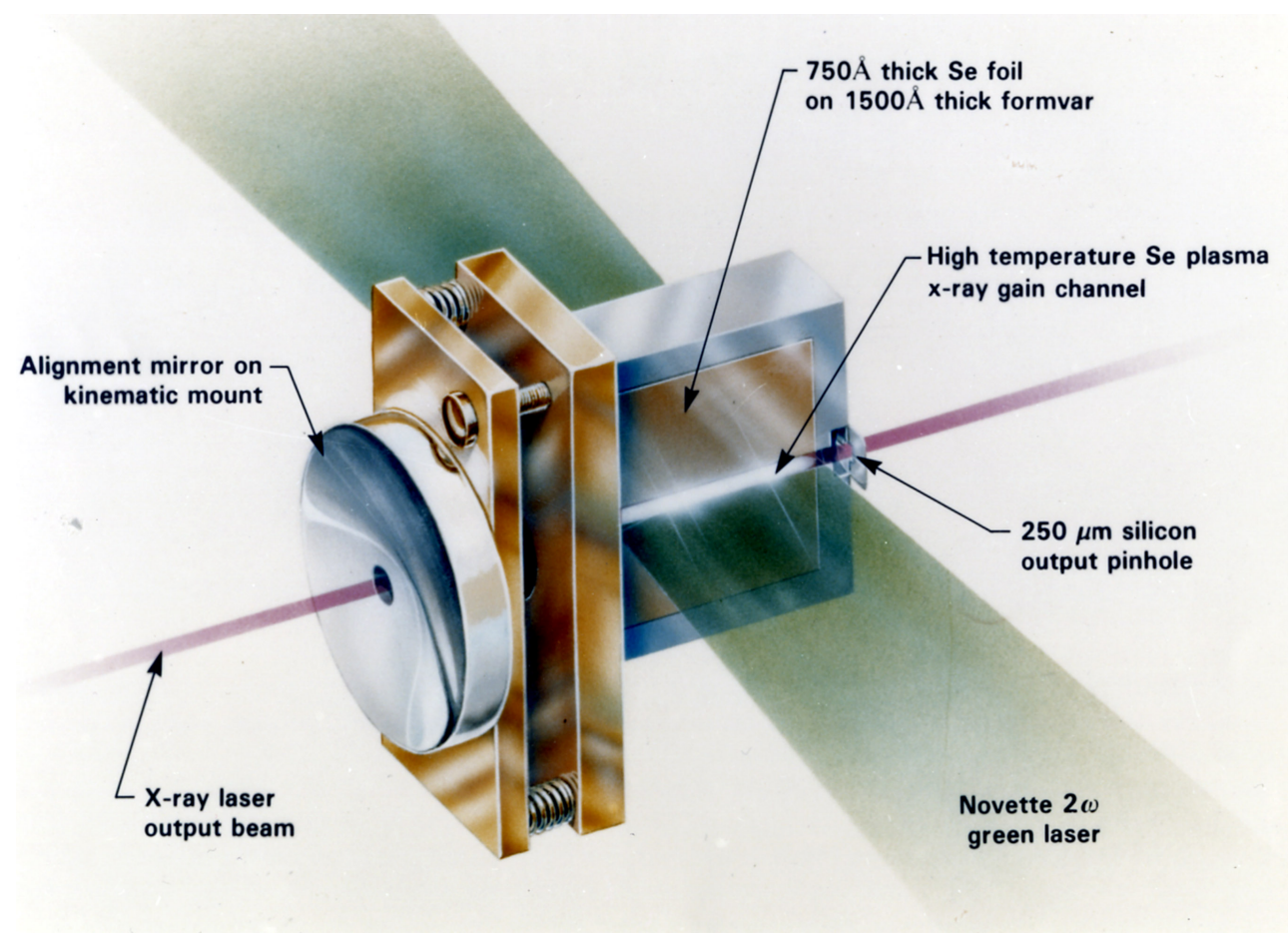

Fig. 2. Schematic of the Nova X-ray laser target showing two line-focused beams illuminating each side of a thin exploding foil target of 75-nm thick selenium coated on a 150-nm thick plastic substrate. Refs. 8 and 9 have more details. 
With the higher energies of the Nova laser the target length was increased and saturated output was achieved in selenium. Over the next decade many neon-like materials lased with the strongest being neon-like yttrium $(\mathrm{Z}=39)$ at 15.5 $\mathrm{nm}$ [17] with an X-ray laser output of $7 \mathrm{~mJ}$ driven by about $5 \mathrm{~kJ}$ of energy from the Nova laser. Other laboratories around the world achieved lasing on neon-like lines in multiple materials [11] with many experiments using germanium $(Z=32)$ because it was lower $\mathrm{Z}$ and easier to achieve lasing at smaller ICF laser facilities.

Designing X-ray lasers required more complex atomic physics models and kinetics codes. Meeting this challenge required greatly improved atomic physics codes such as YODA and kinetics codes such as XRASER. With XRASER one could calculate the gain including detailed radiation transport to model trapping and photo-pumping effects. Today the CRETIN code [18] developed at LLNL is widely used for modeling X-ray laser kinetics and X-ray spectroscopy.

The X-ray laser program required a new level of understanding and measurements of the atomic physics of highly charged ions. The electron-beam ion trap (EBIT) was developed and built at LLNL to meet this need. With EBIT researchers can create any ionization stage of any ion and measure the spectroscopy with unprecedented accuracy.

One initial mystery with the selenium X-ray laser was the absence of lasing on the $3 \mathrm{p}^{1} \mathrm{~S}_{0}-3 \mathrm{~s}{ }^{1} \mathrm{P}_{1}$ line at $18.3 \mathrm{~nm}$ that was predicted to have the largest gain. In the experiments the two strongest laser lines observed were the $3 p^{1} \mathrm{D}_{2}-3 \mathrm{~s}^{3} \mathrm{P}_{1}$ and $3 p{ }^{3} \mathrm{P}_{2}-3 \mathrm{~s}{ }^{1} \mathrm{P}_{1}$ lines which are populated by collisional excitation from the neon-like ground state and by collisional recombination from fluorine-like. The yttrium laser was unique among the neon-like lasers [19] because only one line dominated likely due to the $3 \mathrm{p}^{1} \mathrm{D}_{2}-3 \mathrm{~s}^{3} \mathrm{P}_{1}$ line being nearly resonant with the missing $3 \mathrm{p}{ }^{1} \mathrm{~S}_{0}-3{ }^{1} \mathrm{P}_{1}$ line. This made it better suited for experiments that used X-ray optics that were only optimized for a single wavelength.

Many applications were developed using the $5 \mathrm{~kJ}$ Nova-driven X-ray laser during 1990's. X-ray laser imaging was developed at the Nova laser facility along with greatly improved multi-layer X-ray optics that allowed one to build interferometers and other advanced imaging diagnostics using X-ray lasers as the light source. In addition, a tabletop Xray microscope was developed using an $8 \mathrm{keV} \mathrm{Cu} \mathrm{K-a} \mathrm{source} \mathrm{that} \mathrm{achieved} 10-\mu \mathrm{m}$ spatial resolution using Fresnel zone plates. This legacy continues today as LLNL leads the X-ray optics effort at the SLAC Linac Coherent Light Source (LCLS) facility which operates an X-ray free-electron laser (XFEL) [20].

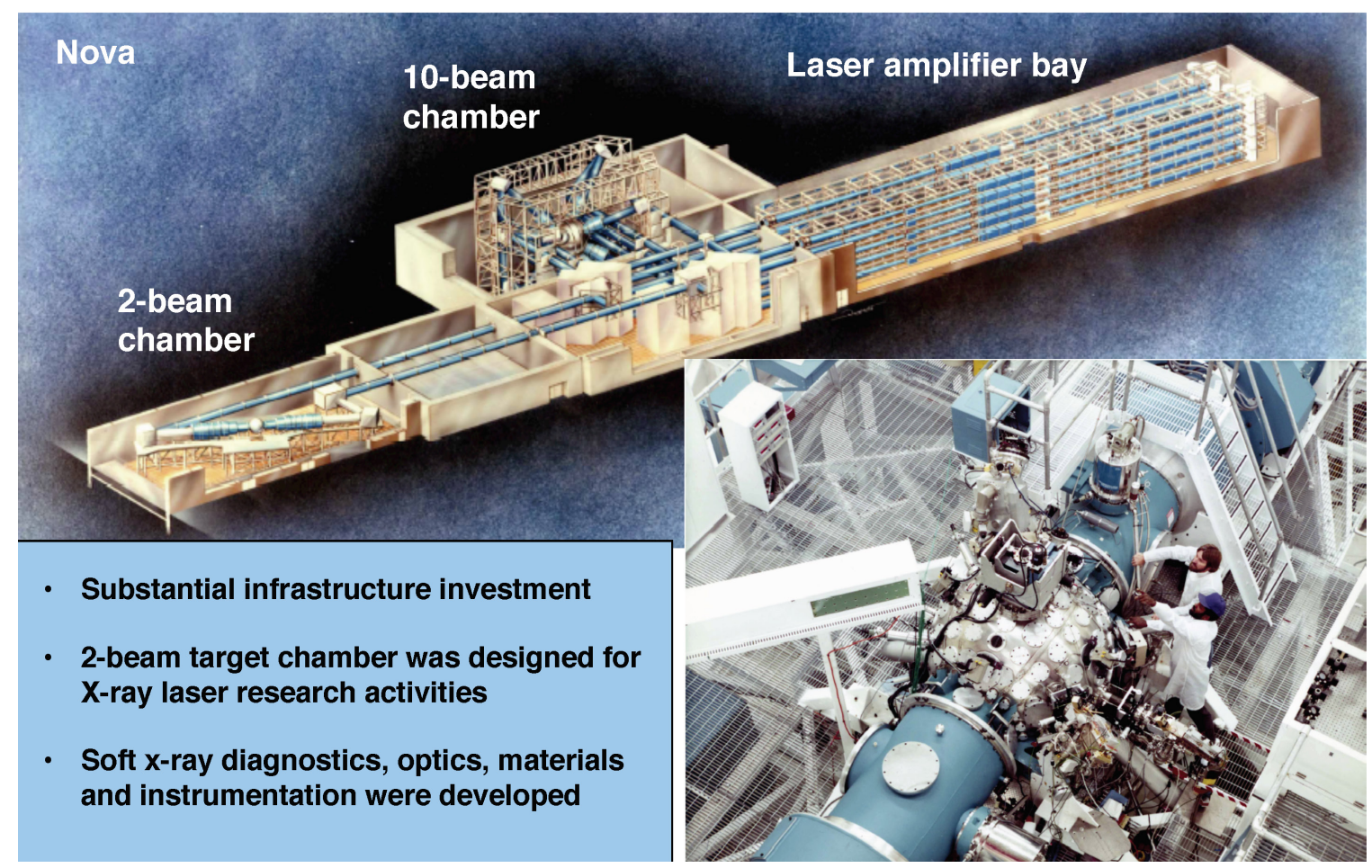

Fig. 3. Schematic of the Nova laser facility with 10 beams going into the main ICF target chamber or 2 beams switched to go into the 2-beam (X-ray laser) target chamber. The photograph shows two people working on the 2-beam target chamber. The X-ray laser targets are illuminated by two counter-propagating, frequency-doubled Nova beams. 
Many advances took place in developing new materials. For example, aerogel technology took a leap forward with the creation of super-light silica aerogels with densities as low as $1 \mathrm{mg}$ per $\mathrm{cc}$ and the production of organic aerogels. There was great interest in building targets at densities below the critical density for laser absorption by the Nova laser. Fig. 4 shows a piece of SEAgel floating on soap bubbles in a beaker. This is very-low-density $(1-300 \mathrm{mg} / \mathrm{cc})$ organic-based foam that is made from the food- thickening agent agar. It is safe enough to eat and could be used as food packaging or encapsulating material for timed- release medication.

In particular, experiments were done to use radiography to image a laser-heated aluminum foil and observe the expansion of the foil [21]. X-ray laser interferometry to measure the two-dimensional electron density of an exploded foil was developed [22] using a Mach-Zehnder configuration. Building the interferometer involved fabricating multi-layer Xray mirrors and X-ray beam-splitters. X-ray microscopy [23] using the nickel-like tantalum X-ray laser at $4.5 \mathrm{~nm}$ was used to do imaging microscopy of a rat sperm cell as shown in Fig. 5. This involved fabricating zone plate objectives as well as normal incidence multi-layer mirrors that operated at $4.5 \mathrm{~nm}$ and was used to observe features as small as $50-\mathrm{nm}$.

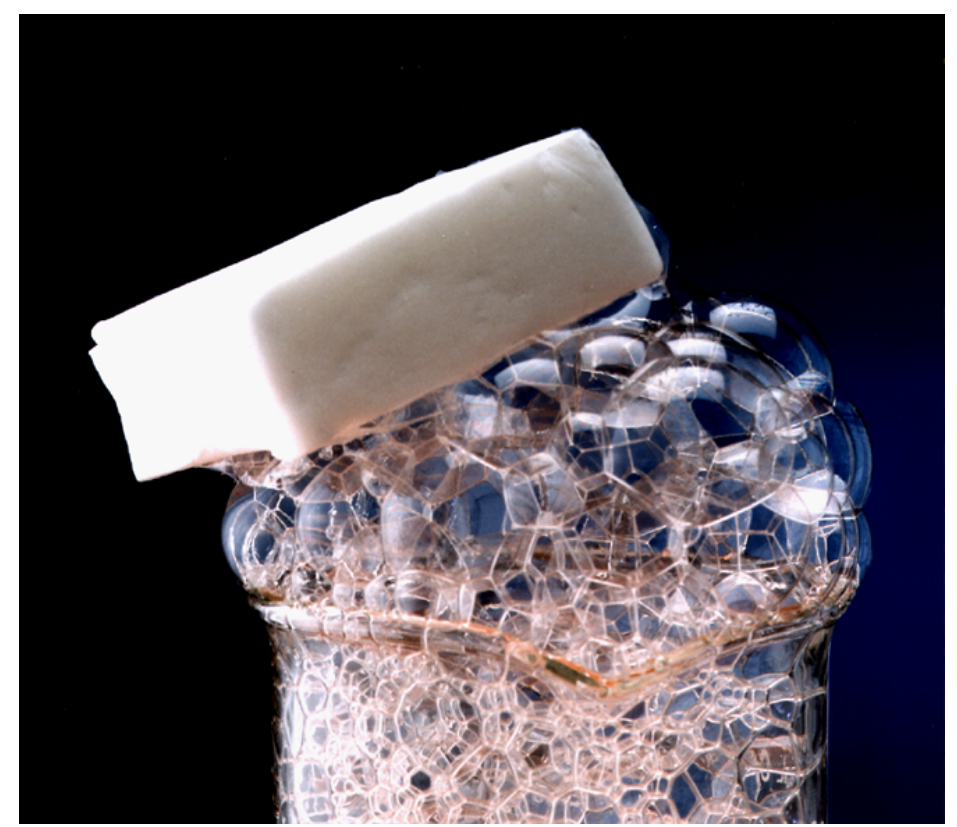

Fig. 4. A piece of SEAgel floating on soap bubbles in a beaker. This very-low-density organic-based foam, made from the food- thickening agent agar, is safe enough to eat and could be used as food packaging or encapsulating material for timed- release medication.

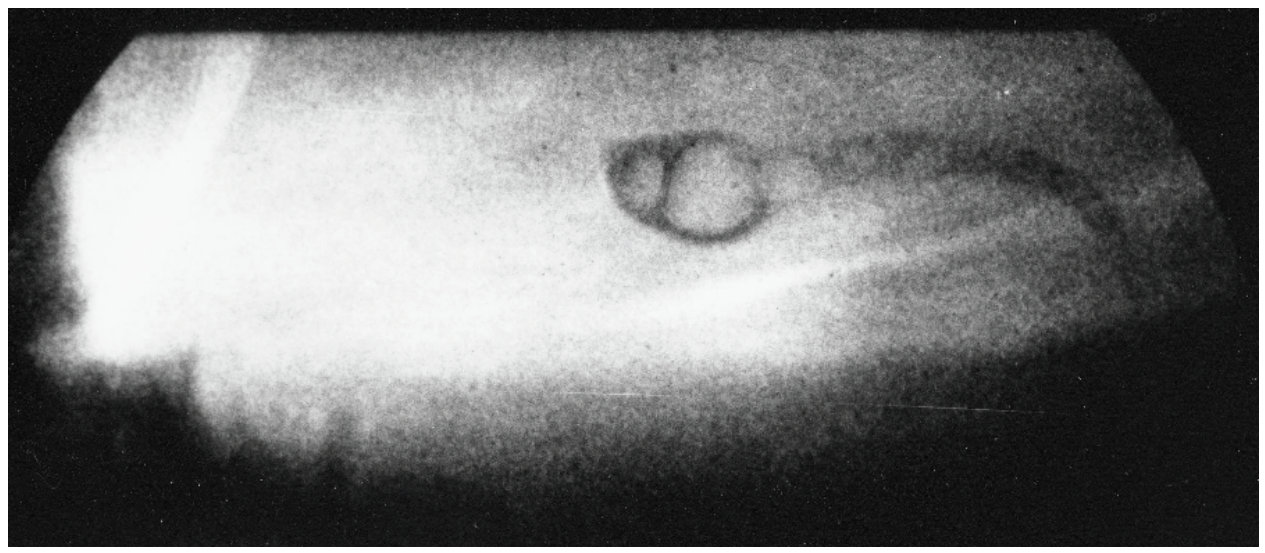

Fig. 5. Image of rat sperm captured using an x-ray microscope that uses the 44.83-nm nickel-like tantalum laser line as the light source. The vertical extent of image is about $10-\mu \mathrm{m}$. This image shows 50-nm features as described in Ref. 23. 
These experiments all helped advance the development of advanced X-ray optics that are used today in many applications. A major goal at the time was to do laser interferometry of biological materials which pushed the development of X-ray lasers to reach shorter wavelengths in the "water window" between 2.3 and $4.4 \mathrm{~nm}$, which are the absorption edges for oxygen and carbon.

To reach shorter wavelengths researchers switched to lasing in nickel like materials. Soon after the success of neonlike selenium, nickel-like europium $(\mathrm{Z}=63)$ lased on the nickel-like $4 \mathrm{~d}^{1} \mathrm{~S}_{0}-4 \mathrm{p}{ }^{1} \mathrm{P}_{1}$ transition at $7.1 \mathrm{~nm}$ with the $4 \mathrm{~d}^{1} \mathrm{~S}_{0}$ upper laser state populated by monopole collisional excitation from the nickel-like ground state [24]. These lasers worked similar to neon-like lasers except they were higher-Z materials based on the closed K, L, and M shell, hence nickel-like (28 electrons). Nickel-like systems have the advantage of producing shorter wavelength lines than neon-like by a factor of two to three for similar excitation energies. The early nickel-like lasers were plagued with very low gain and never achieved saturated output but did reach wavelengths as short as $3.56 \mathrm{~nm}$ in nickel-like gold [25].

\section{TABLE TOP X-RAY LASERS}

In 1992 the big break through on the road to table-top X-ray lasers and in understanding X-ray laser physics was the development of the pre-pulse technique [10] at the Nova laser facility. For the first time strong lasing was observed on the $3 \mathrm{p}^{1} \mathrm{~S}_{0}-3 \mathrm{~s}{ }^{1} \mathrm{P}_{1}$ line in neon-like titanium at $32.6 \mathrm{~nm}$ followed shortly by lasing in chromium at $28.5 \mathrm{~nm}$ and iron at $25.5 \mathrm{~nm}$ as shown in Fig. 6. This line is commonly referred to as the $\mathrm{J}=0-1$ line to distinguish it from the pair of $\mathrm{J}=2-1$ lines seen in the original Se experiments. $J$ is the total angular momentum of the atomic energy level.

With the pre-pulse technique a small $6 \mathrm{~J}, 600$-ps full-width half maximum (FWHM) pulse of energy illuminated a solid slab of titanium and created a plasma. By allowing the plasma to expand for $7 \mathrm{~ns}$ a large uniform plasma was created that

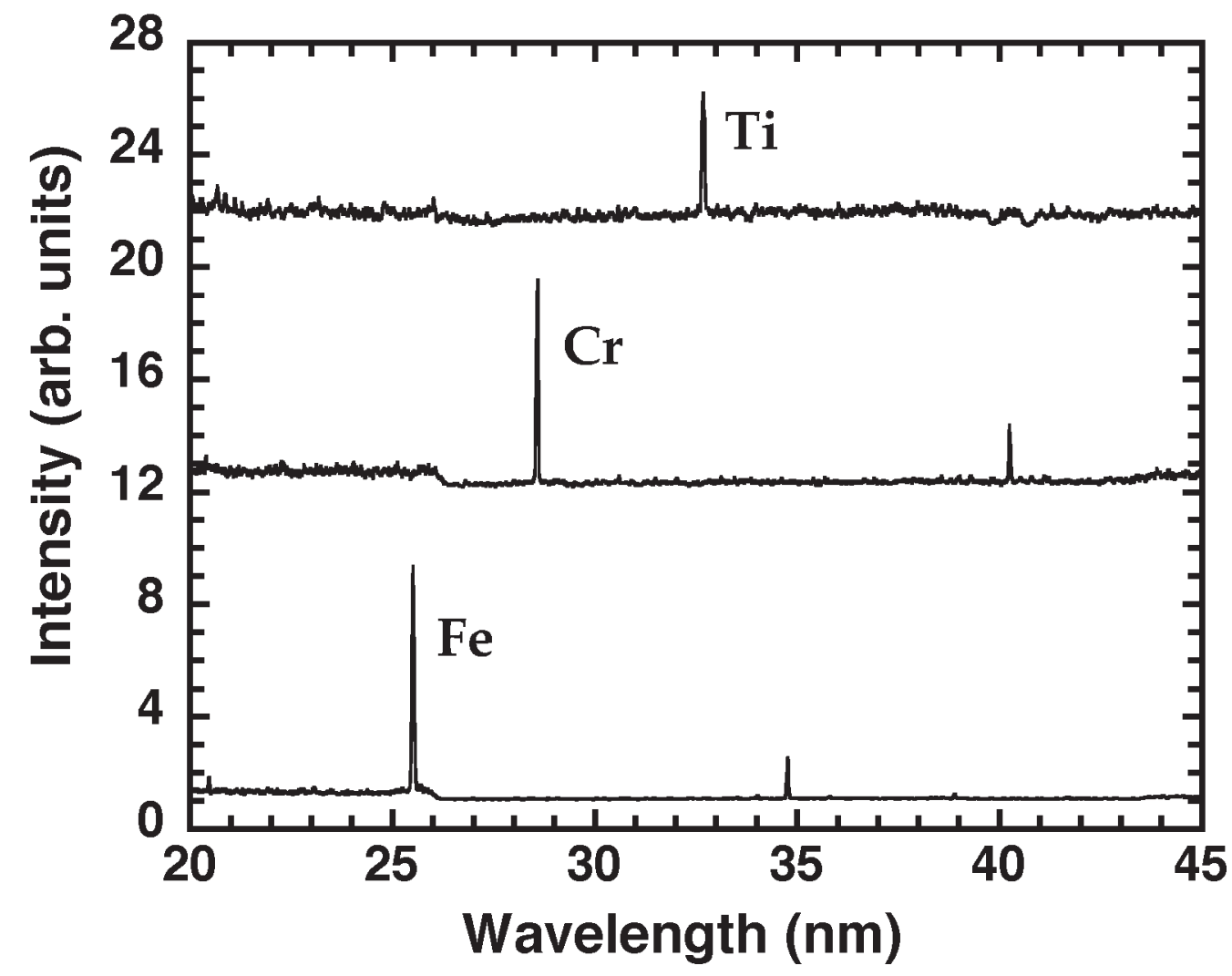

Fig. 6. Spectra of 3.8-cm long Ti, Cr, and Fe targets illuminated by a single $120-\mu \mathrm{m}$ wide line-focused beam of Nova consisting of a $6 \mathrm{~J}$ prepulse followed $7 \mathrm{nsec}$ later by an $1100 \mathrm{~J}$ main pulse. Lasing is observed on the dominant $\mathrm{J}=0-1$ laser lines at 32.6-nm in Ti, 28.5-nm in Ni, and 25.5-nm in Fe. See Ref. 10. Other weaker laser lines are also observed. 
was then illuminated by a $1100 \mathrm{~J}, 600$-ps pulse which heated the titanium to lasing conditions and caused strong lasing on the $32.6 \mathrm{~nm}$ line. The laser was focused to a $120-\mu \mathrm{m}$ wide by $5.4-\mathrm{cm}$ long line focus giving a peak intensity of $3.4 \mathrm{x}$ $10^{13} \mathrm{~W} / \mathrm{cm}^{2}$ on target. The pair of weaker $\mathrm{J}=2-1$ laser lines that had dominated laser output in materials from $\mathrm{Ge}, \mathrm{Se}$, and Y were only weakly observed, if at all. After careful modelling it was shown that the reason lasing had only been seen weakly in the past on the $\mathrm{J}=0$ - 1 line was due to density gradients. Because this line was populated primarily by direct collisional excitation it favored a somewhat higher density region of the plasma which gave it higher gain but subject it to strong refraction effects that made propagation through the gain media more difficult. The use of the pre-pulse created a larger more uniform plasma that allowed the $\mathrm{J}=0-1$ line to propagate well. After independent confirmatory experiments [26] at the Asterix laser, where experiments with neon-like zinc done with and without the pre-pulse conclusively showed the critical role of the pre-pulse, the X-ray laser community switched to using pre-pulses to produce virtually all neonlike X-ray lasers and the $\mathrm{J}=0-1$ line, which had originally been missing for the first eight years, became the standard laser line.

The next big break-through combined the nsec pre-pulse technique to create a uniform plasma with a psec laser pulse to heat the plasma and create gain. These experiments use chirped pulse amplification (CPA) $[27,28]$ to produce the psec duration drive pulse with typical energies less than $10 \mathrm{~J}$. The nsec prepulse is created by using part of the uncompressed beam from the CPA system. Experiments typically use less than $10 \mathrm{~J}$ in each beam. A big advantage of these systems is that they are compact, tabletop, higher-repetition rate systems that occupy a few standard optical benches and they have largely replaced the enormous ICF lasers like Nova used in the past. These systems are also much more affordable and enabled universities to engage in X-ray laser research. Given the short pulse duration these experiments use a travelling

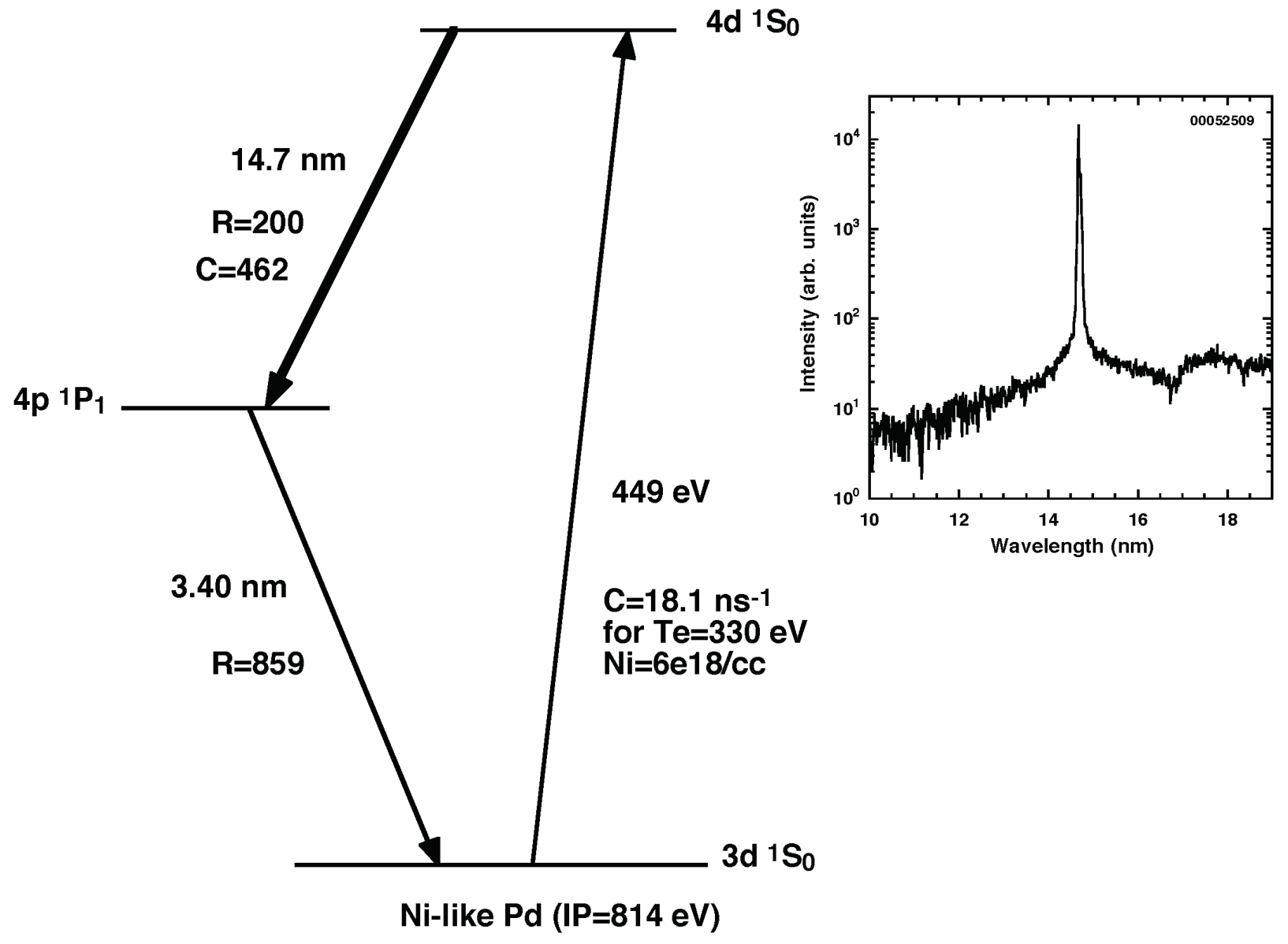

Fig. 7. The nickel-like palladium X-ray laser at $14.7 \mathrm{~nm}$ can be modeled as a 3-level system driven by collisional monopole excitation as shown in this diagram, which is not to scale. The collision (C) rates and radiative (R) rates are shown in nsec ${ }^{-1}$ for a typical plasma heated to $330 \mathrm{eV}$. The spectrum shows strong lasing on the 14.7-nm Ni-like Pd laser line as described in Ref. 30. 
wave geometry to transversely illuminate the $\mathrm{X}$-ray laser target and synchronize the pumping with the lasing process at the speed of light. The shorter psec pulse requires less energy to heat a target to similar temperatures as nsec pulse.

The first experiments [27] of this type in 1995 used a $4 \mathrm{~J}, 1.5 \mathrm{~ns}$ pulse focused to a $30-\mu \mathrm{m}$ by $0.5-\mathrm{cm}$ line focus to illuminate a solid titanium $(Z=22)$ target. This created the pre-plasma that was then heated by a similarly line focused 7 J, $0.7 \mathrm{ps}$ pulse. The titanium lased on two neon-like transitions, the $3 \mathrm{p}{ }^{1} \mathrm{~S}_{0} \rightarrow 3 \mathrm{~s}{ }^{1} \mathrm{P}_{1}$ transition at $32.6 \mathrm{~nm}$ and the $3 \mathrm{~d}^{1} \mathrm{P}_{1}$ $\rightarrow 3 \mathrm{p}{ }^{1} \mathrm{P}_{1}$ transition at $30.1 \mathrm{~nm}$. The second transition at $30.1 \mathrm{~nm}$ was the first self photo-pumped X-ray laser transition that had been observed [29] and was pumped by optical trapping of the strong $2 p{ }^{1} \mathrm{~S}_{0}-3 \mathrm{~d}^{1} \mathrm{P}_{1}$ resonance line.

After the success of the neon-like titanium laser, attention was quickly turned to using the short pulse CPA lasers to drive nickel-like ions. At LLNL we built the COMET laser facility to replicate the facility at the Max-Born Institute. Using the COMET laser nickel-like palladium $(\mathrm{Z}=46)$ lased in 1997 on the $4 \mathrm{~d}^{1} \mathrm{~S}_{0} \rightarrow 4 \mathrm{p}{ }^{1} \mathrm{P}_{1}$ transition at $14.7 \mathrm{~nm}$ [28]. Figure 7 shows the energy level diagram for this laser as well as the collisional and radiative rates for a typical lasing plasma at an electron temperature of $330 \mathrm{eV}$ and ion density of $6 \times 10^{18} \mathrm{~cm}^{-3}$. This corresponds to an electron density of about $1.1 \times 10^{20} \mathrm{~cm}^{-3}$. The $4 \mathrm{~d}^{1} \mathrm{~S}_{0}$ upper laser state is populated by monopole collisional excitation of the nickel-like ground state. The $4 \mathrm{p}^{1} \mathrm{P}_{1}$ lower state decays by rapid radiative decay to the nickel-like ground state. The X-ray laser can be modelled as a 3-level system. Optimizing the drive conditions, palladium reached saturated output using a total of $7 \mathrm{~J}$ from a $1.054-\mu \mathrm{m} \mathrm{Nd}$ :glass laser to drive a $0.9 \mathrm{~cm}$ long target. Typical conditions use a $1 \mathrm{~J}, 600-\mathrm{ps}$ pulse followed $700-$ psec later by a $5 \mathrm{~J}, 1-p s e c$ pulse to illuminate a solid palladium target with a $80-\mu \mathrm{m}$ by $1.1 \mathrm{~cm}$ line focus [30]. A typical spectrum is shown in Fig. 7. Small signal gain coefficients as large as $61 \mathrm{~cm}^{-1}$ were measured with a total gain-length product of 18.1. Typical output observed was $12 \mu \mathrm{J}$ per pulse with divergence of 2 - 3 mrad. The CPA laser system used in these experiment has a repetition rate of one shot every four minutes.

The next big break through in reducing the energy requirements for the $\mathrm{X}$-ray laser was the use of the grazing incidence pumping geometry, commonly referred to as the GRIP geometry [31]. The table-top X-ray lasers of a decade ago used a line-focused psec CPA laser that illuminated the target at normal incidence with a pre-pulse followed by a short main pulse. This geometry is inefficient because the optical laser energy is mainly absorbed at the critical density for the optical light, which is an electron density near $1.7 \times 10^{21} \mathrm{~cm}^{-3}$ for the $0.8 \mu \mathrm{m}$ Ti:sapphire laser drive. Meanwhile the gain region for the X-ray laser typically has an electron density significantly below critical denisty. To improve the efficiency it was realized that illuminating the target with the optical laser at a grazing angle would better couple the optical laser energy into the region of the plasma with X-ray laser gain. This pumping geometry improves the laser coupling efficiency into the gain region of the plasma by using refraction to turn the pump laser at an electron density below the critical density, thus increasing the path length and absorption in this specific region of the plasma. This was first demonstarted with a nickel-like molybdenum target that lases at $18.9 \mathrm{~nm}$ [31] as shown in Fig. 8. For that case the optimum lasing was

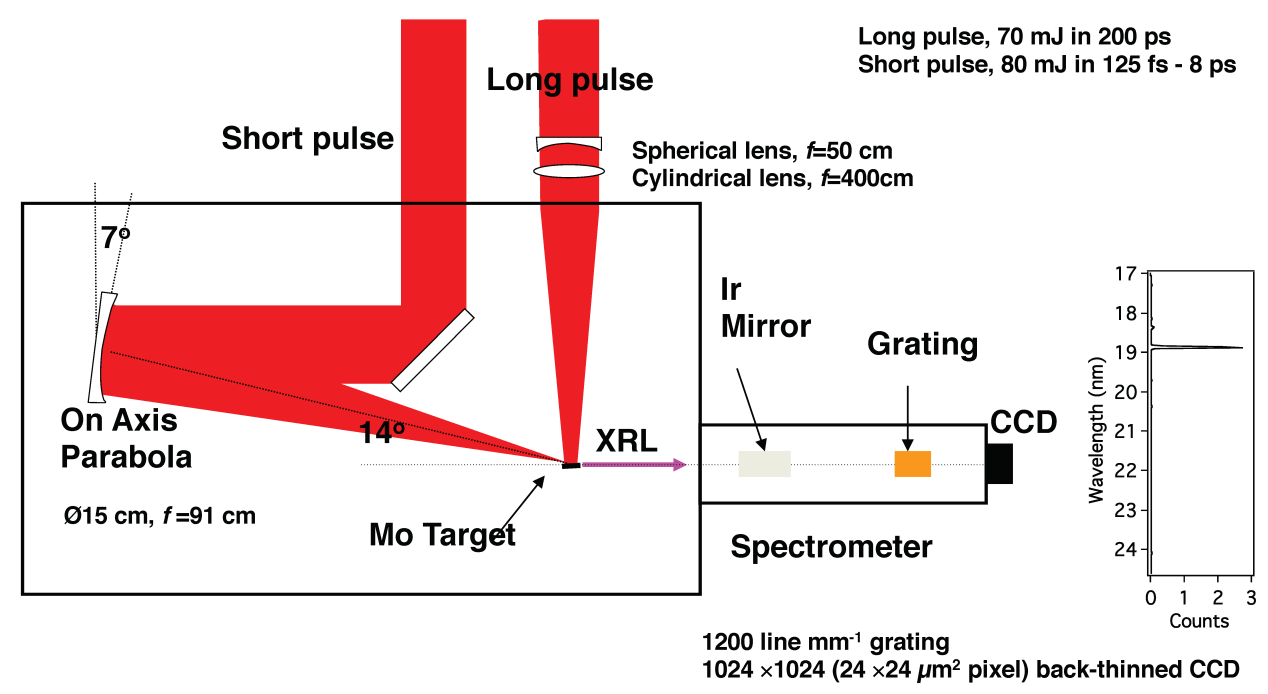

Fig. 8. Schematic of the GRIP geometry showing the $70 \mathrm{~mJ}$ long pulse normally incident on the Mo target followed by the $80 \mathrm{~mJ}$ short pulse that illuminates the target at a grazing angle of 14 degrees to create lasing at $18.9 \mathrm{~nm}$ in Ni-like Mo shown in the spectrum on the right. More details are given in Ref. 31. 
predicted to be at an electron density of $10^{20} \mathrm{~cm}^{-3}$. The optimum grazing angle can be estimated from $\mathrm{n}_{\mathrm{e}}$ (gain) $=\mathrm{n}_{\mathrm{e}}$ (critical) $\sin ^{2}(\phi)$ which gives $\phi=14^{\circ}$ for this case. With this shallow angle one acheives a travelling wave velocity of $\mathrm{c} / \cos (\phi)=$ $1.03 \mathrm{c}$, where $\mathrm{c}$ is the speed of light [32]. It was shown that by tilting the compression grating for the CPA system one could achieve a travelling wave velocity of $1.0 \mathrm{c}$ [33]. The higher efficiency of the grazing incidence (GRIP) pumping geometry requires only a very low energy laser pump $(<150 \mathrm{~mJ})$, available at a $10 \mathrm{~Hz}$ repetition rate, and represents more than an order of magnitude reduction in the pumping energy compared to the conventional transverse scheme.

Improvements in optical lasers has led to the replacement of flashlamps with diodes to create diode-pumped solid state lasers such as the $\mathrm{Yb}: \mathrm{YAG}$ amplifiers that can produce up to $1 \mathrm{~J}$ of output at $100 \mathrm{~Hz}$. This output is compressed using a dielectric grating pair to pulse lengths as short as $5 \mathrm{ps}$. Utilizing the $1.03 \mu \mathrm{m} \mathrm{Yb}$ :YAG laser with $0.9 \mathrm{~J}$ of output at $100 \mathrm{~Hz}$ in the GRIP geometry to drive a 0.5 -cm long silver target enabled the demonstration of a saturated nickel-like silver X-ray laser with $1.0 \mu \mathrm{J}$ of output at $13.9 \mathrm{~nm}$ [34]. With this same system other nickel-like lasers ranging from palladium $(Z=46)$ to tellurium $(Z=52)$ have lased with all reaching saturated output except for the tellurium. Many other laboratories have achieved similar results with wavelengths as short as $6.85 \mathrm{~nm}$ in nickel-like samarium $(Z=62)$ produced using a 10-J drive in the GRIP geometry [35].

\section{CONCLUSIONS}

In this paper we tell the story of the X-ray laser (see also Sect. 1 and Sect. 2 [36]), which began as an attempt to extend optical lasers to shorter wavelengths in the 1970's. Research took off in the 1980's driven by the rivalry between the United States and Soviet Union in their quest to create a "Star Wars" laser shield against ICBM's. At the same time large inertial fusion confinement (ICF) lasers, such as Novette and Nova at LLNL, were able to create the first laboratory X-ray lasers in Ne-like Se at 20.6 and $20.9 \mathrm{~nm}$ using $2 \mathrm{~kJ}$ of energy in a $0.5 \mathrm{~ns}$ pulse. These large ICF lasers could only be fired every few hours and used very thin expensive exploding foils as the targets. With the demonstration of the pre-pulse technique at Nova, where a small pulse of a few joules heated a solid target and create a pre-plasma that was then be heated by a second large pulse to create the lasing conditions, X-ray lasers started working robustly at many laser facilities around the world. The advent of high repetition rate psec lasers combined with the prepulse technique and the grazing incident (GRIP) geometry opened the door to the many table-top X-ray lasers today which can be driven by less than $1 \mathrm{~J}$ of energy and operate at repetition rates at or above $100 \mathrm{~Hz}$. This has opened many new research opportunities for scientific research using X-ray lasers.

Acknowledgements. This work was performed under the auspices of the U.S. Department of Energy by Lawrence Livermore National Laboratory under Contract DE-AC52-07NA27344.

\section{REFERENCES}

[1] Vinogradov, A. V., Sobelman, I. I., Yukov, E. A., "Feasibility of a far ultraviolet laser utilizing transitions of multicharged ions in inhomogeneous plasma," Sov. J. Quantum Electron. 5, 59-63 (1975).

[2] Elton R. C., "Quasi-stationary population inversion on K $\alpha$ transitions," Appl. Opt. 14, 2243- 2249 (1975).

[3] Ilyukhin A. A., Peregudov G. V., Ragozin E. N., Sobel'man I. I., and Chirkov V. A., "Concerning the problem of lasers for the far ultraviolet at approximately $500-700 \AA$,” JETP Lett. 25, No. 12, 535-539 (1977).

[4] Vinogradov A. V., Sobel'man I. I., and Yukov E. A., "Population inversion of transitions in neon-like ions," Sov. J. Quantum Electron. 7, 32 - 35 (1977).

[5] Elton R. C., [X-ray Lasers] Academic, San Diego, CA (1990).

[6] Chapline G. and Wood L., "X-ray lasers, "Physics Today, pp. 40 - 48 (June 1975).

[7] Suckewer S., Skinner C. H., Milchberg H., Keane C., and Voorhees D., "Amplification of stimulated soft-x-ray emission in a confined plasma column," Phys. Rev. Lett. 55, 1753 - 1756 (1985).

[8] Matthews D. L., Hagelstein P. L., Rosen M. D., Eckart M. J., Ceglio N. M., Hazi A. U., Medecki H., MacGowan B. J., Trebes J. E., Whitten B. L., Campbell E. M., Hatcher C. W., Hawryluk A. M., Kauffman R. L., Pleasance L. D., Rambach G., Scofield J. H., Stone G., and Weaver T. A., "Demonstration of a soft x-ray amplifier," Phys. Rev. Lett. $54,110-113$ (1985). 
[9] Rosen M. D., Hagelstein P. L., Matthews D. L., Campbell E. M., Hazi A. U., Whitten B. L., MacGowan B. J., Turner R. E., Lee R. W., Charatis G., Busch Gar. E., Shepard C. L., and Rockett P. D., "Exploding-foil technique for achieving a soft-X-ray laser,” Phys. Rev. Lett. 54, 106-109 (1985).

[10] Nilsen J., MacGowan B. J., Da Silva L. B., and Moreno J. C., "Prepulse technique for producing low-Z Ne-like Xray lasers," Phys. Rev. A 48, 4682 - 4685 (1993).

[11] Daido H., "Review of soft x-ray laser researches and development," Rep. Prog. Phys. 65, 1513-1576 (2002).

[12] Clarence A. Robinson, Jr., "Advance made on high-energy laser," Aviation Week \& Space Technology, pp. 25-27 (February 23, 1981).

[13] Nilsen J., "Legacy of the X-ray Laser Program," Lawrence Livermore National Laboratory report UCRL-LR-114552, $<$ https://www.osti.gov/biblio/10182456-legacy-ray-laser-program> (August 6, 1993).

[14] Nilsen J., "Legacy of the X-ray Laser Program," Lawrence Livermore National Laboratory report UCRL-52000-9411 in Energy \& Technology Review p. 13-21, <https://str.1lnl.gov/content/pages/past-issues-pdfs/1994.11.pdf $>$ (November 1994). Also see $<$ https://www.osti.gov/biblio/10123059-energy-technology-review-november $>$

[15] Nilsen, J., "X-ray lasers: the evolution from Star Wars to the table-top," Lawrence Livermore National Laboratory report LLNL-PRES-817183, <https://doi.org/10.2172/1759988> (December 2020). Summary and link also available at $<$ https://www.osti.gov/biblio/1759988/>

[16] Avrorin E. N., Lykov V. A., Loboda P. A., and Politov V. Yu., "Review of theoretical works on X-ray laser research performed at RFNC-VNIITF," Laser and Particle Beams 15, 3 -15 (1997).

[17] DaSilva L. B., MacGowan B. J., Mrowka S., Koch J. A., London R. A., Matthews D. L., and Underwood J. H., "Power measurements of a saturated yttrium x-ray laser," Opt. Lett. 18, 1174-1176 (1993).

[18] Scott, H. A., "Cretin - a radiative transfer capability for laboratory plasmas," JQSRT 71, 689-701, 2001.

[19] Nilsen J. and Scofield J. H., "Wavelengths of neon-like 3p - 3s X-ray laser transitions," Physica Scripta 49, 588 - 591 (1994).

[20] SLAC National Accelerator Laboratory $<$ http://lcls.slac.stanford.edu/ $>$

[21] Cauble R., DaSilva L. B., Barbee Jr. T. W., Celliers P., Moreno J. C., and Wan A. S., "Micron-resolution radiography of laser-accelerated and X-ray heated foils with an X-ray laser," Phys. Rev. Lett. 74, 3816 -3819 (1995).

[22] DaSilva L. B., Barbee Jr. T. W., Cauble R., Celliers P., Ciarlo D., Libby S. B., London R. A., Matthews D. L., Mrowka S., Moreno J. C., Ress D., Trebes J. E., Moreno J. C., and Weber F., "Electron density measurements of high denisty plasmas using soft X-ray laser interferometry," Phys. Rev. Lett. 74, 3991-3994 (1995).

[23] DaSilva L. B., Trebes J. E., Balhorn R., Mrowka S., Anderson E., Attwood D. T., Barbee Jr. T. W., Brase J., Corzett M., Gray J., Koch J. A., Lee C., London R. A., MacGowan B. J., Matthews D. L., and Stone G., "X-ray laser microscopy of rat sperm nuclei," Science 258, 269 - 271 (1992).

[24] MacGowan B. J., Maxon S., Hagelstein P. L., Keane C. J., London R. A., Matthews D. L., Rosen M. D., Scofield J. H., and Whelan D. A., "Demonstration of soft X-ray amplication in nickel-like ions," Phys. Rev. Lett. 59, 2157-2160 (1987).

[25] MacGowan B. J., DaSilva L. B., Fields D. J., Keane C. J., Koch J. A., London R. A., Matthews D. L., Maxon S., Mrowka S., Osterheld A. L., Scofield J. H., Shimkaveg G., Trebes J. E., and Walling R. S., "Short wavelength x-ray laser research at the Lawrence Livermore National Laboratory," Phys. Fluids B 4, 2326 - 2337 (1992).

[26] Fill E. E., Li Y. L., Schlögl D., Steingruber J., and Nilsen J., "Sensitivity of lasing in neon-like zinc at $21.2 \mathrm{~nm}$ to the use of the prepulse technique," Opt. Lett. 20, 374 - 376 (1995).

[27] Nickles P. V., Shlyaptsev V. N., Kalachnikov M., Schnürer M., Will I., and Sandner W., "Short Pulse X-Ray Laser at $32.6 \mathrm{~nm}$ Based on Transient Gain in Ne-like Titanium," Phys. Rev. Lett. 78, 2748-2751 (1997).

[28] Dunn J., Osterheld A. L., Shepherd R., White W. E., Shlyaptsev V. N., and Stewart R. E., "Demonstration of X-Ray Amplification in Transient Gain Nickel-like Palladium Scheme," Phys. Rev. Lett. 80, 2825-2828 (1998).

[29] Nilsen J., Fiedorowicz H., Bartnik A., Li Y. L., Lu P. X., and Fill E. E., "Self photo-pumped neon-like X-ray laser," Opt. Lett. 21, 408 - 410 (1996).

[30] Dunn J., Li Y. L., Osterheld A. L., Nilsen J., Hunter J. R., and Shlyaptsev V. N., "Gain saturation regime for laserdriven tabletop, transient Ni-like ion x-ray lasers," Phys. Rev. Lett. 84, $4834-4837$ (2000).

[31] Keenan, R., Dunn, J., Patel, P. K., Price, D. F., Smith, R. F., Shlyaptsev, V. N., "High-Repetition-Rate GrazingIncidence Pumped X-Ray Laser Operating at 18.9 nm," Phys. Rev. Lett. 94, 103901 (2005).

[32] Bleiner, D., Feurer, T., Springer Proceedings in Physics Volume 147 - X-Ray Lasers 2012, 35-38 (2014).

[33] Chanteloup, J.-C., Salmon, E., Sauteret, C., Migus, A., Zeitoun, P., Klisnick, A., Carillon, A., Hubert, S., Ros, D., Nickles, P., Kalachnikov, M., J., "Pulse-front control of 15-TW pulses with a tilted compressor, and application to the subpicosecond traveling-wave pumping of a soft-x-ray laser,” Opt. Soc. Am. B 17, 151-157 (2000). 
[34] Reagan, B. A., Berrill, M., Wernsing, K. A., Baumgarten, C., Woolston, M., Rocca, J. J., "High-average-power, 100Hz-repetition-rate, tabletop soft-x-ray lasers at sub-15-nm wavelengths," Phys. Rev. A 89, 053820 (2014).

[35] Balmer, J., Staub, F., Jia, F., "Soft-X-Ray lasing down to $6.85 \mathrm{~nm}$ in Ni-like samarium," Springer Proceedings in Physics Volume 147 - X-Ray Lasers 2012, 29-34 (2014).

[36] D. Bleiner, The Science and Technology of X-ray Lasers: A 2020 Update Proc. SPIE 11886, 1188602 (2021) 